\title{
Refusal of viral testing during the SARS-CoV-2 pandemic
}

\author{
Authors: John H McDermott ${ }^{A}$ and William G Newman ${ }^{B}$
}

\begin{abstract}
Widespread testing for the respiratory syndrome coronavirus-2 (SARS-CoV-2) will represent an important part of any strategy designed to safely reopen societies from lockdown. Healthcare settings have the potential to become reservoirs of infectivity, and therefore many hospital trusts are beginning to carry out routine screening of staff and patients. This could promote the effective cohorting of patients and reduce the rate of nosocomial infection. However, for various reasons, some individuals may refuse this testing. Here we highlight this as an emergent ethicolegal issue which we expect to become increasingly relevant as testing becomes ubiquitous. We explore this position from an ethical and legal perspective, determining whether refusal of testing is acceptable under UK law. Individual patients refusing testing could undermine a hospital's testing strategy; therefore clinicians and policy makers must prospectively determine the best course of action if this were to occur.
\end{abstract}

KEYWORDS: Testing, COVID-19, SARS-CoV-2, ethics

DOI: $10.7861 /$ clinmed.2020-0388

\section{Introduction}

The severe acute respiratory syndrome coronavirus-2 (SARS-CoV-2) pandemic has caused a dramatic shift in the way we live our lives and a significant reduction in the level of routine healthcare activity. Governments around the world are now beginning to grapple with how to safely reopen their societies and restart regular healthcare services. It is now widely accepted, through lessons learnt from the 2003 SARS outbreak, that extensive testing (antibody and/or antigen) will form a major part in any strategy. To ensure the effective isolation of infected individuals and reduce nosocomial spread, widespread testing of health and care staff and patients will be necessary.

In many hospitals, all inpatient admissions are now being tested for SARS-CoV-2, informing cohorting strategies. Anecdotally, however, colleagues have encountered patients who have

Authors: ${ }^{A}$ NIHR academic clinical fellow in genomic medicine and clinical genetics SpR, Manchester Centre for Genomic Medicine, Manchester, UK and University of Manchester, Manchester, UK; ${ }^{B}$ professor of translational genomic medicine and consultant clinical geneticist, Manchester Centre for Genomic Medicine, Manchester, UK and University of Manchester, Manchester, UK refused to undergo antigen testing, via a nasal swab. At the time of presentation these individuals did not have overt signs of COVID-19, but testing was recommended as part of the hospital infection control policy. In the cases we are aware of, the health professionals accepted the patients' decisions, no specific reasons were given for refusal and testing was not pursued. As a result the question arises of whether, in the context of the current pandemic, that refusal is ethically or legally acceptable.

\section{Individual autonomy versus public interest}

Patient autonomy is a key pillar of medical ethics. In exceptional circumstances autonomy is overridden by competing interests, for example if there is a public interest in protecting others from a serious communicable disease. SARS-CoV-2 is a serious communicable disease and the Coronavirus Act 2020 sets out requirements for individuals 'at such times as the public health officer may specify to provide a biological sample, or to allow a healthcare professional to take a biological sample by appropriate means.' ${ }^{2}$ Further, the Act states that 'it is an offence in a case where a person is directed, to fail without reasonable excuse to comply with the direction.' Failure to comply could result in a maximum fine of $£ 1,000 .{ }^{3}$ These powers are similar to those regarding notifiable diseases set out in the existing Public Health (Control of Disease) Act 1984.

Should individuals be able to exert their autonomy, without sanction, in the context of the risks to others due to coronavirus? Clinical practice will dictate that untested individuals are treated as if they are infected with COVID-19. Staff will have to use higher levels of personal protective equipment (PPE) when undertaking clinical interactions or procedures. The individual may be placing themselves at increased risk as they will potentially be cared for with patients known to be affected with COVID-19. An individual who refuses testing and then is infected may also have a claim in negligence against his/her health professional that they had not used the powers given to them by the Coronavirus Act.

Health professionals and care workers are increasingly being tested for SARS-CoV-2 to reduce risks of nosocomial infection and to inform the need for isolation. ${ }^{4}$ It should be noted that the law does not differentiate between patients and staff, who may also be obliged to undergo testing if they have been in contact with an infected patient or are potentially infected themselves, which many asymptomatic health professionals will be. ${ }^{4}$

\section{Lessons from HIV and tuberculosis}

The concept of non-compliance is not novel to the SARS-CoV-2 pandemic and there has been rigorous ethical debate around 
this concept in the past for other infectious diseases. The most commonly cited example is where a patient refuses HIV testing after a health professional suffers a needlestick injury. ${ }^{5}$ In this situation the patient's refusal is harmful to that individual health professional, exposing them to weeks of post-exposure prophylaxis (PEP), psychological distress, and potential viral infection. In this scenario, there is no legal mechanism to compel the patient to provide a sample against an autonomous refusal and to do so would be a breach of the Mental Capacity Act (2005) and possibly the Human Tissue Act (2004).

The risk of HIV transmission following a needlestick injury is relatively low, approximately $0.13 \%$, and although PEP can have significant side effects, it represents an effective management option. ${ }^{6}$ This is not the case in the context of a patient with possible COVID-19. Here the patient is at high risk of passing on their infection to multiple individuals and, unlike following a needlestick injury, there are no definitive management options. A more similar scenario might be the refusal of testing for tuberculosis (TB), another highly transmissible and potentially fatal respiratory disorder. It has previously been argued that patients should be notified about testing and given the opportunity to object. ${ }^{7}$ If an objection is raised, the burden should be on those proposing coercive testing to show that the expected public health benefits are sufficient to justify overriding the individual's choice. This more closely parallels the current testing paradigm in COVID-19, where there is a clear and overriding public health benefit to testing.

Although testing for SARS-CoV-2 and HIV cannot be directly compared, the HIV testing guidelines provide mature and considered advice regarding patient refusal which are translatable. The 2009 Royal College of Physicians guidelines for HIV testing state that 'if a patient refuses a test, the reasons why they have made that choice should be explored to ensure that these are not due to incorrect beliefs about the virus or the consequences of testing. ${ }^{8}$ Although COVID-19 does not have the same historical stigmatisation attached to it as HIV may do, some patients may have misconceptions about the virus which can be addressed through discussion.

\section{Conclusion}

Health professionals should emphasise the consequences of an individual's refusal to be tested and try to persuade them, rather than invoking the law. However, it is important to note that the law is in place as a last option if required, although it is difficult to imagine a situation where a hospital would seek to involve the authorities for a non-compliant patient or staff member. Rather, as inpatient and staff screening becomes more prevalent, hospitals and other healthcare facilities should consider how best as institutions they might respond to an individual refusing a test. Best practice guidelines could be drafted clarifying the optimal approach to discussing this with patients and staff members. We argue that this would not need to represent an entirely new body of work; rather it should derive from the existing literature around testing for other communicable diseases such as HIV and TB.

\section{Funding}

WGN is supported by the Manchester NIHR BRC (ISBRC-121520007). JHM is supported by the NIHR ACF Programme.

\section{References}

1 Cheng VC, Chan JF, To KK, Yuen KY. Clinical management and infection control of SARS: lessons learned. Antiviral Res 2013;100: 407-19.

2 Government of the United Kingdom. Coronavirus Act 2020. Schedule 21: Powers relating to potentially infectious persons. www.legislation.gov.uk/ukpga/2020/7/schedule/21/enacted.

3 Knapton S, Mikhailova A. Public face $£ 1,000$ fine or custody for refusing coronavirus tests and quarantine. Daily Telegraph, 15 March 2020

4 Black JRM, Bailey C, Swanton C. COVID-19: the case for health-care worker screening to prevent hospital transmission. Lancet 2020; 395:1418-20.

5 Grime PR. Testing without consent. Occup Med (Lond) 2013;63: 84-6.

6 Baggaley RF, Boily MC, White RG, Alary M. Risk of HIV-1 transmission for parenteral exposure and blood transfusion: a systematic review and meta-analysis. AIDS 2006;20:805-12.

7 Coleman CH, Selgelid MJ, Reis A, Reichman LB, Jaramillo E. The role of informed consent in tuberculosis testing and screening. Eur Respir ] 2012;39:1057-9.

8 Palfreeman A, Fisher M, Ong E et al. Testing for HIV: concise guidance. Clin Med 2009;9:471-6.

Address for correspondence: Dr John H McDermott, Manchester Centre for Genomic Medicine, Manchester University Hospitals NHS Foundation Trust, Manchester M13 9WL, UK.

Email: john.mcdermott2@mft.nhs.uk 